Article

\title{
Definite Integral of Arctangent and Polylogarithmic Functions Expressed as a Series
}

\author{
Robert Reynolds*(D) and Allan Stauffer \\ Department of Mathematics and Statistics, York University, Toronto, ON M3J 1P3, Canada \\ * Correspondence: milver@my.yorku.ca; Tel.: +1-(416)-319-8383
}

Received: 13 October 2019; Accepted: 12 November 2019; Published: 14 November 2019

\begin{abstract}
We present a method using contour integration to evaluate the definite integral of arctangent reciprocal logarithmic integrals in terms of infinite sums. In a similar manner, we evaluate the definite integral involving the polylogarithmic function $L i_{k}(y)$ in terms of special functions. In various cases, these generalizations give the value of known mathematical constants such as Catalan's constant $G$, Aprey's constant $\zeta(3)$, the Glaisher-Kinkelin constant $A, \log (2)$, and $\pi$.
\end{abstract}

Keywords: Catalan; zeta; logarithm tangent function; Lerch function; polylogarithmic function; Glaisher-Kinkelin constant; contour integration

\section{Introduction}

We use our new method to evaluate definite integrals in the form of a series [1]. This is a novel approach to these problems in mathematics and has not been used before to our knowledge. This method involves using a form of the Cauchy integral formula. Both the definite integral and infinite sum can be written in terms of the same contour integral, and therefore, we can equate the two. We will evaluate integrals of the form $\frac{1}{y} \arctan (y) \log ^{k}(a y)$ where $R e(k)<-1$ as well as $\frac{\log ^{k}(a y)}{y} L i_{m}(c y)$ over $y \in[0, \infty)$ in the form of known functions. The parameters in these integrals are complex in general.

\section{Results}

\subsection{The Contour Integral}

We start with Cauchy's integral Formula (1) where $C$ is the generalized Hankel contour as described by Reynolds and Stauffer [1].

$$
\frac{y^{k}}{k !}=\frac{1}{2 \pi i} \int_{C} \frac{e^{w y}}{w^{k+1}} d w
$$

\subsection{The Arctangent Reciprical Logarithmic Integral}

We replace $y$ by $\log (a y)$ and multiply both sides by $\frac{1}{y} \arctan (y)$ in (1) to get the Cauchy equation

$$
\frac{\arctan (y) \log ^{k}(a y)}{y k !}=\frac{1}{2 \pi i} \int_{C} \frac{\arctan (y)(a y)^{w}}{y w^{k+1}} d w,
$$

where the definition of the logarithmic function is from Section 4.1 in [2]. This definition of the logarithmic function is used throughout this paper. Then, we take the definite integral over $y \in[0, \infty)$ of both sides to get 


$$
\begin{aligned}
\int_{0}^{\infty} \frac{\arctan (y) \log ^{k}(a y)}{y k !} d y & =\frac{1}{2 \pi i} \int_{0}^{\infty} \int_{C} \frac{\arctan (y)(a y)^{w}}{y w^{k+1}} d w d y \\
& =\frac{1}{2 \pi i} \int_{C} \int_{0}^{\infty} \frac{\arctan (y)(a y)^{w}}{y w^{k+1}} d y d w \\
& =-\frac{1}{4 i} \int_{C} a^{w} w^{-k-2} \sec \left(\frac{\pi w}{2}\right) d w
\end{aligned}
$$

from $\int_{0}^{\infty} x^{\alpha-1} \arctan (x) d x=-\frac{\pi}{2 \alpha \cos (\alpha \pi / 2)}$, Equation (2.7.4.7) in [3], where the integral is valid for $-1<\operatorname{Re}(w)<0$ and $\operatorname{Re}(k)<-1$

\subsection{Infinite Sum of the Contour Integral}

In this section, we will derive an equivalent contour integral by taking an infinite sum over $n$ of a transformed Cauchy integral formula. We once again start with Equation (1), multiplying both sides by $(-1)^{n+1} \pi$, replacing $y$ with $\pi i(2 n+1) / 2+\log (a)$ and $k$ with $k+1$ to yield

$$
\frac{(-1)^{n+1} \pi(\pi i(2 n+1) / 2+\log (a))^{k+1}}{(k+1) !}=\frac{(-1)^{n+1} \pi}{2 \pi i} \int_{C} \frac{e^{w(\pi i(2 n+1) / 2+\log (a))}}{w^{k+2}} d w .
$$

We then take the infinite sum of both sides over $[0, \infty)$ with $\operatorname{Re}(k)<-1$ to get

$$
\begin{aligned}
\sum_{n=0}^{\infty} \frac{(-1)^{n+1} \pi(\pi i(2 n+1) / 2+\log (a))^{k+1}}{(k+1) !} & =\sum_{n=0}^{\infty} \frac{(-1)^{n+1} \pi}{2 \pi i} \int_{C} \frac{e^{w(\pi i(2 n+1) / 2+\log (a))}}{w^{k+2}} d w \\
& =\frac{1}{2 i} \int_{C} \sum_{n=0}^{\infty}(-1)^{n+1} \frac{e^{w(\pi i(2 n+1) / 2+\log (a))}}{w^{k+2}} d w \\
& =-\frac{1}{4 i} \int_{C} a^{w} w^{-k-2} \sec \left(\frac{\pi w}{2}\right) d w
\end{aligned}
$$

from $\operatorname{sech}(x)=2 \sum_{k=0}^{\infty}(-1)^{k} e^{-(2 k+1) x}$, Equation (1.232.2) in [4], where $\operatorname{sech}(i x)=\sec (x)$ and $\operatorname{Im}(w)>0$ for the sum to converge.

\subsection{Equating the Definite Integral and Infinite Sum}

Since the right-hand side of Equations (3) and (5) are equal, we can equate the left-hand sides of these equations and simplify the factorials to yield

$$
\int_{0}^{\infty} \frac{\arctan (y)}{y} \log ^{k}(a y) d y=-\frac{\pi}{k+1} \sum_{n=0}^{\infty}(-1)^{n}(\pi i(2 n+1) / 2+\log (a))^{k+1},
$$

where $\operatorname{Re}(k)<-1$. If $a$ is real and positive, then ay will be unity for some finite value of $y$, which implies that $\log (a y)$ will be zero and the integrand will diverge at that point. However, the series converges as long as the real part of $k$ is negative. Thus, the principal value of the integral will be given by the value of the series.

\subsection{Evaluations in Terms of Fundamental Constants}

Here we will look at a few examples of the integral in Equation (6), which yields new integral forms in terms of constants such as Catalan's constant $\zeta$ and $\pi$. 
(1) When $k=-2$. Replace $a$ with $e^{a i}$

$$
\begin{aligned}
\int_{0}^{\infty} \frac{\arctan (y)}{y \log ^{2}\left(e^{a i} y\right)} d y & =\pi \sum_{n=0}^{\infty} \frac{(-1)^{n}}{\pi i(2 n+1) / 2+\log \left(e^{a i}\right)} \\
& =-i \sum_{n=0}^{\infty} \frac{(-1)^{n}}{n+1 / 2+a / \pi)} \\
& =-i \Phi\left(-1,1, \frac{1}{2}+\frac{a}{\pi}\right)
\end{aligned}
$$

from $\Phi(z, s, v)=\sum_{n=0}^{\infty}(v+n)^{-s} z^{n}$ where $|z|<1, v \neq 0,-1, \ldots$, Equation (9.550.1) in [4], and $\Phi$ is the Lerch function. We will now simplify the left-hand side by rationalizing the denominator to get

$$
\begin{aligned}
\int_{0}^{\infty} \frac{\arctan (y)}{y} \frac{d y}{(a i+\log (y))^{2}}= & \int_{0}^{\infty} \frac{\arctan (y)}{y} \frac{-2 a i \log (y)}{\left(\log ^{2}(y)+a^{2}\right)^{2}} d y+ \\
& \int_{0}^{\infty} \frac{\arctan (y)}{y} \frac{\left(\log ^{2}(y)-a^{2}\right)}{\left(\left(\log ^{2}(y)+a^{2}\right)^{2}\right.} d y
\end{aligned}
$$

Now, comparing the real and imaginary coefficients on the left-hand sides of Equations (7) and (8),

$$
\int_{0}^{\infty} \frac{\arctan (y)\left(\log ^{2}(y)-a^{2}\right)}{y\left(\log ^{2}(y)+a^{2}\right)^{2}} d y=0 .
$$

This result can be shown by breaking the integral into two parts, integrating from $y \in[0,1]$ and $y \in[1, \infty)$. In the latter, replace $y$ by $1 / y$ and use the log form of the arctan function to show that the two integrals are the negatives of one another and hence zero. We also have

$$
\int_{0}^{\infty} \frac{\arctan (y) \log (y)}{y\left(\log ^{2}(y)+a^{2}\right)^{2}} d y=\frac{1}{2 a} \Phi\left(-1,1, \frac{1}{2}+\frac{a}{\pi}\right) .
$$

(2) When $k=-2$ and $a=-1$. Note That $\log (-1)=\pi i$.

$$
\begin{aligned}
\int_{0}^{\infty} \frac{\arctan (y)}{y} \frac{d y}{\log ^{2}(-y)} & =\pi \sum_{n=0}^{\infty} \frac{(-1)^{n}}{\pi i(2 n+1) / 2+\log (-1)} \\
& =-i \sum_{n=0}^{\infty} \frac{(-1)^{n}}{n+3 / 2} \\
& =-i \frac{4-\pi}{2}
\end{aligned}
$$

from $\sum_{k=1}^{\infty}(-1)^{k+1} \frac{1}{2 k-1}=\frac{\pi}{4}$, Equation (0.232.2) in [4]. We will now simplify the left-hand side by rationalizing the denominator to get

$$
\begin{aligned}
\int_{0}^{\infty} \frac{\arctan (y)}{y} \frac{d y}{(\log (-1)+\log (y))^{2}}=\int_{0}^{\infty} \frac{\arctan (y)}{y} \frac{-2 i \pi \log (y)}{\left(\log ^{2}(y)+\pi^{2}\right)^{2}} d y+ \\
\int_{0}^{\infty} \frac{\arctan (y)}{y} \frac{\left(\log ^{2}(y)-\pi^{2}\right)}{\left(\log ^{2}(y)+\pi^{2}\right)^{2}} d y
\end{aligned}
$$

Now, comparing the real and imaginary coefficients on the left-hand sides of Equations (11) and (12),

$$
\int_{0}^{\infty} \frac{\arctan (y)\left(\log ^{2}(y)-\pi^{2}\right)}{y\left(\log ^{2}(y)+\pi^{2}\right)^{2}} d y=0
$$


from the procedure used in (9), and

$$
\int_{0}^{\infty} \frac{\arctan (y) \log (y)}{y\left(\log ^{2}(y)+\pi^{2}\right)^{2}} d y=\frac{4-\pi}{4 \pi}
$$

(3) When $k=-2$ and $a=i$. Note That $\log (i)=\pi i / 2$.

$$
\begin{aligned}
\int_{0}^{\infty} \frac{\arctan (y)}{y} \frac{d y}{\log ^{2}(i y)} & =\pi \sum_{n=0}^{\infty} \frac{(-1)^{n}}{\pi i(2 n+1) / 2+\log (i)} \\
& =-i \sum_{n=0}^{\infty} \frac{(-1)^{n}}{n+1} \\
& =-i \log (2)
\end{aligned}
$$

from $\sum_{k=1}^{\infty}(-1)^{k+1} \frac{1}{k}=\log (2)$, Equation (0.232.1) in [4]. We will now simplify the left-hand side by rationalizing the denominator to get

$$
\begin{aligned}
\int_{0}^{\infty} \frac{\arctan (y)}{y} \frac{d y}{(\log (i)+\log (y))^{2}}=\int_{0}^{\infty} \frac{\arctan (y)}{y} \frac{-i \pi \log (y)}{\left(\log ^{2}(y)+\pi^{2} / 4\right)^{2}} d y+ \\
\\
\int_{0}^{\infty} \frac{\arctan (y)}{y} \frac{\log ^{2}(y)-\pi^{2} / 4}{\left(\log ^{2}(y)+\pi^{2} / 4\right)^{2}} d y
\end{aligned}
$$

Now, comparing the real and imaginary coefficients on the left-hand sides of Equations (15) and (16),

$$
\int_{0}^{\infty} \frac{\arctan (y)\left(\log ^{2}(y)-\pi^{2} / 4\right)}{y\left(\log ^{2}(y)+\pi^{2} / 4\right)^{2}} d y=0
$$

from the procedure used in (9), and

$$
\int_{0}^{\infty} \frac{\arctan (y) \log (y)}{y\left(\log ^{2}(y)+\pi^{2} / 4\right)^{2}} d y=\frac{\log (2)}{\pi} .
$$

\section{Table of Integrals}

We can use other values of $k$ and generate more integrals, in particular, starting from Equation (6) for numerical values of $k$ and $a$, the entries in the Table 1 ,

Table 1. Table of Integrals.

\begin{tabular}{llc}
\hline$k$ & $a$ & $\int_{0}^{\infty} \frac{\arctan (y)}{y} \log ^{k}(a y) d y$ \\
\hline-3 & -1 & $2 \frac{(G-1)}{\pi}$ \\
-3 & $i$ & $-\frac{\pi}{24}$ \\
-3 & $e^{a i}$ & $\frac{1}{8 \pi}\left(\zeta\left(2, \frac{1}{2}\left(1+\frac{2 a+\pi}{2 \pi}\right)-\zeta\left(2, \frac{2 a+\pi}{4 \pi}\right)\right)\right.$ \\
\hline
\end{tabular}

where $\operatorname{Re}(a)>0, \mathrm{G}$ is Catalan's constant, and $\zeta$ is the Hurwitz zeta function given by (9.521.1) in [4]. 


\section{Integrals Involving the Polylogarithmic Function}

\subsection{Definite Integral of the Contour Integral}

We replace $y$ by $\log (a y)$ in (1) and multiply both sides by $\frac{L i_{m}(-c y)}{y}$ to get the Cauchy equation

$$
\frac{1}{k !} \frac{\log ^{k}(a y) L i_{m}(-c y)}{y}=\frac{1}{2 \pi i} \int_{C} y^{w-1} L i_{m}(-c y) \frac{a^{w} d w}{w^{k+1}}
$$

Then we take the definite integral over $y \in[0, \infty)$ of both sides to get

$$
\begin{aligned}
\frac{1}{k !} \int_{0}^{\infty} \frac{\log ^{k}(a y) L i_{m}(-c y)}{y} d y & =\frac{1}{2 \pi i} \int_{0}^{\infty} \int_{C} y^{w-1} L i_{m}(-c y) \frac{a^{w} d w d y}{w^{k+1}} \\
& =\frac{1}{2 \pi i} \int_{C}\left(\int_{0}^{\infty} y^{w-1} L i_{m}(-c y) d y\right) \frac{a^{w} d w}{w^{k+1}} \\
& =\frac{(-1)^{m}}{2 i} \int_{C} a^{w} c^{-w} w^{-k-m-1} \csc (\pi w) d w
\end{aligned}
$$

from $\int_{0}^{\infty} x^{\alpha-1} L i_{n}(-c x) d x=\frac{(-\alpha)^{-n} \pi}{\sin (\alpha \pi)} c^{-\alpha}$, Equation (2.5.2.1) in [5], where the integral is valid for $-1<\operatorname{Re}(w)<0,-\pi<\arg (a) \leq \pi$ and $|\arg (c)|<\pi$.

\subsection{Infinite Sum of the Contour Integral}

In this section, we will derive an equivalent contour integral by taking an infinite sum over $n$ of a transformed Cauchy integral formula. We once again start with Equation (1), multiplying both sides by $-2(-1)^{m} \pi i$, replacing $y$ with $\pi i(2 n+1)+\log (a)-\log (c)$ and $k$ with $k+m$ to yield

$$
\frac{-2(-1)^{m} \pi i(\pi i(2 n+1)+\log (a)-\log (c))^{k+m}}{(k+m) !}=\frac{-2(-1)^{m} \pi i}{2 \pi i} \int_{C} \frac{e^{w(\pi i(2 n+1)+\log (a)-\log (c))}}{w^{k+m+1}} d w .
$$

We then take the infinite sum of both sides over $[0, \infty)$ to get

$$
\begin{aligned}
\frac{-2(-1)^{m} \pi i}{(k+m) !} \sum_{n=0}^{\infty}(\pi i(2 n+1)+\log (a)-\log (c))^{k+m} & =(-1)^{m+1} \sum_{n=0}^{\infty} \int_{C} \frac{e^{w(\pi i(2 n+1)+\log (a)-\log (c))}}{w^{k+m+1}} d w \\
& =(-1)^{m+1} \int_{C} \sum_{n=0}^{\infty} \frac{e^{w(\pi i(2 n+1)+\log (a)-\log (c))}}{w^{k+m+1}} d w \\
& =\frac{(-1)^{m}}{2 i} \int_{C} a^{w} C^{-w} w^{-k-m-1} \csc (\pi w) d w
\end{aligned}
$$

from $\csc (x)=2 \sum_{k=0}^{\infty} e^{-(2 k+1) x},(1.232 .3)$ in [4], where $\csc (i x)=-i \csc (x)$ and $\operatorname{Im}(w)>0$ for the sum to converge.

\subsection{Equating the Definite Integral and Infinite Sum}

Since the right-hand sides of Equations (3) and (5) are equal, we can equate the left-hand sides of these equations to yield

$$
\int_{0}^{\infty} \frac{\log ^{k}(a y) L i_{m}(-c y)}{y} d y=\frac{2 \pi i(-1)^{m+1} k !}{(k+m) !} \sum_{n=0}^{\infty}(\pi i(2 n+1)+\log (a)-\log (c))^{k+m}
$$

where $\operatorname{Re}(k+m)<-1, a \neq 0$ and $c \neq 0$. We can simplify the infinite sum on the right-hand side to get

$$
\int_{0}^{\infty} \frac{\log ^{k}(a y) L i_{m}(-c y)}{y} d y=\frac{(-1)^{m+1} k !}{(k+m) !}(2 \pi i)^{k+m+1} \zeta\left(-k-m, \frac{1}{2}+\frac{\log (a)-\log (c)}{2 \pi i}\right)
$$

from $\zeta(z, q)=\sum_{n=0}^{\infty} \frac{1}{(q+n)^{z}}$ where $\operatorname{Re}(z)>1, q \neq 0,-1,-2, \ldots$, (9.521.1) in [4], where $\zeta(r, s)$ is the Hurwitz zeta function, which has a meromorphic continuation in the $r$-plane, its only singularity in $\mathbb{C}$ 
being a simple pole at $r=1$ with residue -1 . Note the left-hand side of Equation (24) converges for all finite $k$. We also note that $\zeta(x, 1)$ is the Riemann zeta function $\zeta(x)$.

\section{Special Cases of the Polylogarithmic Integral}

In this section, we will use the Hurwitz zeta function in Equation (24) to look at values of the parameters $k, a, n$, and $c$, which yield known constants and special functions such as $\zeta(3)$, Aprey's constant, and Catalan's constant $G$.

(1) From Equation (24), when $a=-c$,

$$
(-1)^{m+1} \frac{k !}{(k+m) !}(2 \pi i)^{k+m+1} \zeta(-k-m) .
$$

Choosing particular values of $k$ and $m$, we can obtain $\zeta(2), \zeta(3)$, etc.

(2) From Equation (24), when $a=i c$ and $m=-1$, we get

$$
k(2 \pi i)^{k} \zeta\left(1-k, \frac{3}{4}\right) .
$$

From the above, when $k=-1$,

$$
\frac{1}{2 \pi i}\left(\pi^{2}-8 C\right)
$$

from $\psi^{n}(z)=(-1)^{n+1} n ! \sum_{k=0}^{\infty}(z+k)^{-n-1}$, where $z \neq 0,-1,-2, \ldots,(6.4 .10)$ in [2], where the value of the polygamma function $\psi_{1}(3 / 4)$ is from [6] and $C$ is Catalan's constant.

(3) Similarly, when $a=-i c$ and $m=-1$, we get

$$
k(2 \pi i)^{k} \zeta\left(1-k, \frac{1}{4}\right) .
$$

From the above, when $k=-1$,

$$
\frac{1}{2 \pi i}\left(\pi^{2}+8 C\right)
$$

from Equation (6.4.10) in [2], where the polygamma function $\psi_{1}(1 / 4)$ is from [6] and $C$ is Catalan's constant.

Integral Representation of the Glaisher-Kinkelin Constant

Using Equation (24) and setting $a=-c, m=-1$ and replacing $k$ with $1-k$,

$$
(1-k)(2 \pi i)^{1-k} \zeta(k) \text {. }
$$

Take the derivative of the function with respect to $k$ to get

$$
-(2 \pi i)^{1-k} \zeta(k)-(1-k)(2 \pi i)^{1-k} \log (2 \pi i) \zeta(k)+(1-k)(2 \pi i)^{1-k} \zeta^{\prime}(k) .
$$

Then setting $k=-1$,

$$
\begin{aligned}
& -(2 \pi i)^{2} \zeta(-1)-2(2 \pi i)^{2} \log (2 \pi i) \zeta(-1)+2(2 \pi i)^{2} \zeta^{\prime}(-1) \\
& =-(2 \pi i)^{2}\left(-\frac{1}{12}\right)-2(2 \pi i)^{2} \log (2 \pi i)\left(-\frac{1}{12}\right)+2(2 \pi i)^{2} \zeta^{\prime}(-1) \\
& =-\frac{\pi^{2}}{3}-\frac{2}{3} \pi^{2} \log (2 \pi i)-8 \pi^{2}\left(\frac{1}{12}-\log (A)\right)
\end{aligned}
$$


from $A=e^{\left(1 / 12-\zeta^{\prime}(-1)\right)}$, Equation (2.15) in [7], where $A$ is the Glaisher-Kinkelin constant and from (9.535.2) in [4] and 23.2.24 in [2]. The value of $\zeta(-1)$ is given in [7].

\section{Summary and Future Research Directions}

In Section 1, we presented a novel method to derive a generalized formula for the definite integral over $y \in[0, \infty)$ of $\frac{1}{y} \arctan (y) \log ^{k}(a y)$ where $a$ and $k$ are complex with $\operatorname{Re}(k)<-1$. In Section 2 , we motivated this work by evaluating this integral to known constant forms. We gave several examples involving $\pi, \log (2)$, known as Mercator's constant and Catalan's constant $(G)$, and more are possible using the method presented. We derived similar formulas for definite integrals of the form $\frac{\log ^{k}(a y)}{y} L i_{m}(c y)$ over $y \in[0, \infty)$ in Section 4 , and we expressed a closed form solution for this integral in terms of the Hurwitz zeta function. In Section 5, we evaluated the integral using particular values of the parameter to yield special constants such as Catalan's constant, with more constants possible using the $\zeta$ function. The results in this article were numerically verified for various values, both real and imaginary, of the parameters in the integrals using Mathematica by Wolfram.

Author Contributions: Writing-review and editing, original draft preparation, conceptualization, R.R.; Writing-review and editing, supervision, funding acquisition, A.S.

Funding: This research was supported by NSERC Canada under Grant No. 504070.

Acknowledgments: The authors are grateful to both the referees and the editor for their useful comments and suggestions, which have greatly helped to improve the presentation of this paper.

Conflicts of Interest: The authors declare no conflict of interest.

\section{References}

1. Reynolds, R.; Stauffer, A. Reciprocal Logarithmic Integrals Expressed as a Series. arXiv 2019, arXiv:1906.04927.

2. Abramowitz, M.; Stegun, I.A. (Eds.) Handbook of Mathematical Functions with Formulas, Graphs, and Mathematical Tables, 9th ed.; Dover: New York, NY, USA, 1982.

3. Prudnikov, A.P.; Brychkov, Y.A.; Marichev, O.I. Integrals and Series, Elementary Function; USSR Academy of Sciences: Moscow, Russia, 1986; Volume 1.

4. Gradshteyn, I.S.; Ryzhik, I.M. Tables of Integrals, Series and Products, 6th ed.; Academic Press: Cambridge, MA, USA, 2000.

5. Prudnikov, A.P.; Brychkov, Y.A.; Marichev, O.I. Integrals and Series, More Special Functions; USSR Academy of Sciences: Moscow, Russia, 1990; Volume 3.

6. Kolbig, K.S. The Polygamma Function $\psi_{k}(x)$ for $x=1 / 4$ and $x=3 / 4$. J. Comp. Appl. Math. 1996, 75, 43-46. [CrossRef]

7. Finch, S.R. Encyclopedia of Mathematics and its Applications: Mathematical Constants; Cambridge University Press: Cambridge, UK, 2003.

(C) 2019 by the authors. Licensee MDPI, Basel, Switzerland. This article is an open access article distributed under the terms and conditions of the Creative Commons Attribution (CC BY) license (http://creativecommons.org/licenses/by/4.0/). 